\title{
A rare case of ulcerative proctitis associated with type $B$ lymphomatoid papulosis and superimposed human cytomegalovirus infection
}

\author{
Valeria Criscuoli ${ }^{\mathrm{I}}$, MD, PhD, Maria Rosa Rizzuto ${ }^{2}, \mathrm{MD}$, and Mario Cottone ${ }^{\mathrm{I}}$, MD
}

\author{
${ }^{1}$ Dibimis (Biomedical Department of \\ Internal and Specialistic Medicine), \\ "V.Cervello" Hospital Palermo, \\ University of Palermo, Palermo, Italy, \\ and ${ }^{2}$ Institute of Pathology. "V.Cervello" \\ Hospital Palermo, Palermo, Italy \\ Correspondence \\ Valeria Criscuoli, MD, PhD \\ Via Imperatore Federico 70 \\ 90143 \\ Palermo \\ Italy \\ E-mail: vale.cic@tiscali.it
}

Conflicts of interest: None.

Lymphomatoid papulosis (LyP) is a disorder described by Macaulay $^{\mathrm{I}}$ in I968 as a continuous self-healing, papular eruption, clinically benign, and histologically malignant. The disorder is characterized by erythematous papules and nodules that progress to form vesicular, crusted, or hemorrhagic lesions and then undergo spontaneous healing with scarring. Lesions usually occur over the trunk and limbs. LyP is usually a benign cutaneous disease with alarming histopathologic features; previously believed to be an inflammatory process, it is now included among the cutaneous T-cell lymphomas in the current European Organization for Research and Treatment of Cancer and World Health Organization classifications. ${ }^{2}$

Histopathologically, three types can be distinguished: type A exhibits wedge-shaped lymphocytic infiltrates with abundant Reed-Sternberg-like, $\mathrm{CD}_{3} \mathrm{O}(\mathrm{Ki}-\mathrm{I})$ positive cells mixed with neutrophils and eosinophils; type $B$ shows lymphocytes with smaller, hyperchromatic, cerebriform nuclei predominating and fewer or absent $\mathrm{CD}_{3}$ o staining but with marked epidermal involvement; type $C$ displays a monotonous population of $\mathrm{CD}_{3}$ o-positive cells.

We describe a case of human cytomegalovirus (HCMV) associated ulcerative proctitis diagnosed with LyP type B. A 38-year-old man with a three-month history of rectal bowel disease referral center in June 2008. One month before, in May 2008, he underwent a proctoscopy that showed rectal ulcers $2 \mathrm{~cm}$ wide. He also underwent a biopsy, which revealed a histological description of ulcerative proctitis with evidence of large endothelial cells containing a basophilic intranuclear inclusion $(8-\mathrm{IO} \mu \mathrm{m})$ eccentrically placed with positivity for HCMV on immunohistochemical assay. The immunocytochemical search for HCMV antigenemia pp65 in peripheral leukocytes was also positive.

The other laboratory studies were normal, and his medical history was unremarkable, specifically without any complaint that could be related to bowel disease or immunosuppressive state. The patient had not received any immunomodulant treatment before the referral.

At about the same time, the patient reported the appearance of erythematous papules over the trunk, some of which evolved into crusted lesions. A skin biopsy showed a diagnosis compatible with type B (lymphocytic type) LyP (Figs. I and 2). The patient was treated successfully with topical mesalazine for the ulcerative proctitis and with psoralen-ultraviolet A with gradual improvement and disappearance of the eruptions after eight weeks. No antiviral therapy was dispensed for HCMV infection due to the rapid clinical improvement of 


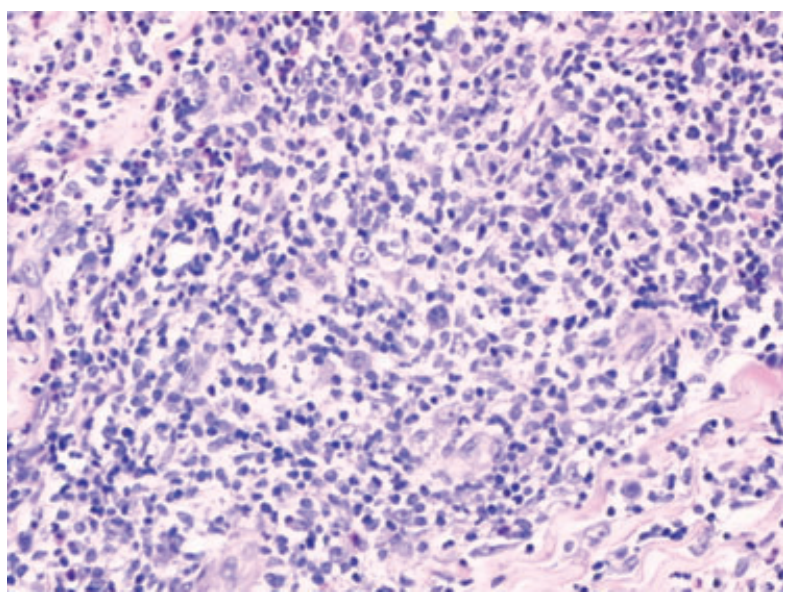

Figure 1 Diffuse dermal-hypodermal infiltrate (consisting of lymphocytes, eosinophils, and histiocytes) with atypical lymphoid cells. Hematoxylin and eosin immunostaining $\times 20$

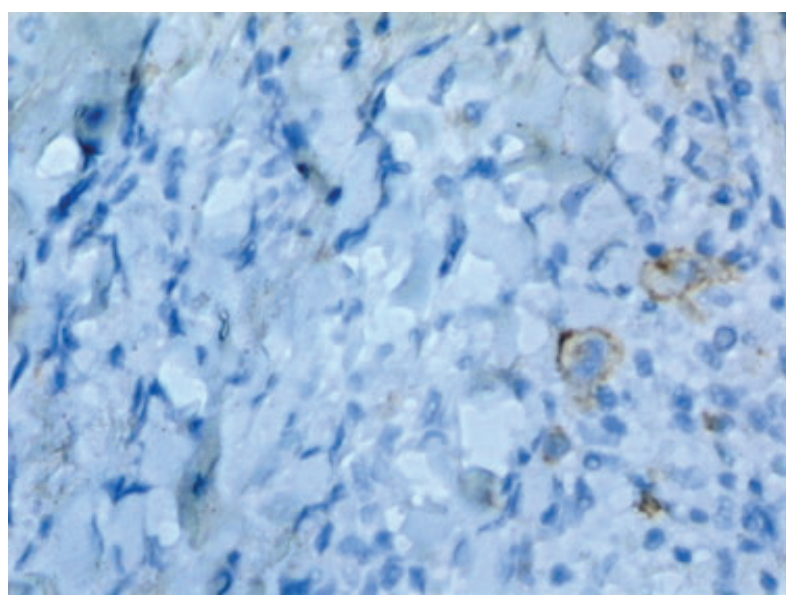

Figure 2 Dense infiltrate in the dermis with $\mathrm{CD}_{30} 0^{+}$large atypical lymphocytes mixed with small $\mathrm{CD}_{3} 0^{-}$lymphocytes. $\mathrm{CD}_{30} \mathrm{O}^{+}$immunostaining, $\times 40$

the patient. In August 2008, the patient underwent total colonoscopy that confirmed only rectal involvement with no extension of disease and with initial recovery of ulcers and histological HCMV disappearance.

The patient visited the hospital as an outpatient every six months. In July 20I0, the patient was in stable clinical remission with topical mesalazine treatment with no other cutaneous manifestations. As a curative therapy is not available for LyP and none of the available treatment modalities affect the natural course of the disease, the short-term benefits of active treatment should be balanced carefully against the potential side effects. Low-dose oral methotrexate $(5-20 \mathrm{mg} /$ week $)$ is the most effective therapy to suppress the development of new skin lesions. Beneficial effects of psoralen-ultraviolet A and topical chemotherapy have been reported. However, after discontinuation of treatment, the disease generally relapses within weeks or months. Therefore, in patients with relatively few and non-scarring lesions, long-term follow-up without active treatment should be considered. ${ }^{3}$ In IO-20\% of patients, it is preceded by, concurrent with, or transforms into malignant lymphoma, most often anaplastic large cell (CD 30 -positive) lymphoma, mycosis fungoides, Hodgkin's disease, or non-Hodgkin's lymphoma of the lymph nodes. No differences in the overall clinical course have been linked to these histological subsets. ${ }^{4}$ To our knowledge, there are no reports concerning the association between HCMV and LyP, particularly in patients with inflammatory bowel disease. Several studies have suggested a role for herpesvirus infection in super-antigenic activation of $\mathrm{T}$ lymphocytes, mainly in other variants of cutaneous T-cell lymphomas such as mycosis fungoides and Sézary syndrome. Two reports show that latent HCMV infection may play a role in the susceptibility of mycosis fungoides in predisposed subjects by inducing $\mathrm{T}$-cell proliferation and resistance to apoptosis; more than $97 \%$ of patients with any at all stages of cutaneous $\mathrm{T}$-cell lymphoma have evidence for previous infection with human HCMV. ${ }^{5,6}$

\section{References}

I Macaulay WL. Lymphomatoid papulosis. A continuing self-healing eruption, clinically benign-histologically malignant. Arch Dermatol I968; 97: 23-30.

2 Willemze R, Kerl H, Sterry W, et al. EORTC classification for primary cutaneous lymphomas: a proposal from the Cutaneous Lymphoma Study Group of the European Organization for Research and Treatment of Cancer. Blood I997; 90: 354-37I.

3 Bekkenk M, Geelen FAMJ, van Voorst Vader PC, et al. Primary and secondary cutaneous $\mathrm{CD}_{3}$ o-positive lymphoproliferative disorders: long term follow-up data of 2I9 patients and guidelines for diagnosis and treatment: a report from the Dutch Cutaneous Lymphoma Group. Blood 2000; 95: 3653-366I.

4 Willemze R, Meyer CJ, Van Vloten WA, Scheffer E. The clinical and histological spectrum of lymphomatoid papulosis. Br J Dermatol I982; I07: I3 I-I44.

5 Herne KL, Talpur R, Breuer-McHam J, et al. Cytomegalovirus seropositivity is significantly associated with mycosis fungoides and Sézary syndrome. Blood 2003; IOI: 2I32-2I36.

6 Ballanger F, Bressollette C, Volteau C, et al. Cytomegalovirus: its potential role in the development of cutaneous T-cell lymphoma. Exp Dermatol 2009; I8: $574-576$. 